\title{
Linfoma T cutáneo en paciente con artritis reumatoidea
}

\author{
Eduardo Mussano', Laura Onetti', Isaac Cadile ${ }^{1}$, María Victoria Gazzoni', María Inés Stancich ${ }^{1}$, María Carolina \\ Taboada Bonfanti ${ }^{1}$, Raúl Jurado ${ }^{1}$, Marina Werner ${ }^{1}$, Rosana Truchia ${ }^{2}$, Marco Mazzotta ${ }^{3}$, Carlos Consigli ${ }^{4}$ \\ ${ }^{1}$ Servicio de Reumatología, ${ }^{2}$ Servicio de Infectología, ${ }^{3}$ Servicio de Anatomía Patológica, ${ }^{4}$ Servicio de Dermatología. Hospital Nacional de Clínicas, \\ Facultad de Ciencias Médicas, Universidad Nacional de Córdoba, Argentina
}

\begin{abstract}
Resumen
La artritis reumatoidea incrementa el riesgo de muerte en pacientes que la padecen, ya sea por las comorbilidades como enfermedades cardiovasculares y las infecciones, como así también por una mayor frecuencia de desórdenes linfoproliferativos asociados a la enfermedad de base. Se presenta el caso de un paciente de sexo masculino de 74 años de edad, con artritis reumatoidea poliarticular y nodular de larga data. Tratado con DMARs, etanercept y abatacept con poca eficacia, por lo que inició rituximab con excelente respuesta, recibiendo luego como mantenimiento tofacitinib $5 \mathrm{mg} /$ día. En abril de este año presenta lesiones nodulares y eritematosas en ambos miembros inferiores que luego se necrotizan y esfacelan. Se suspende tratamiento inmunosupresor. Se indica biopsia de piel que muestra linfoma T cutáneo con compromiso dérmico e hipodérmico. Se inició quimioterapia CHOP pero luego del segundo ciclo el paciente presenta aplasia medular y fallece.
\end{abstract}

Palabras clave: artritis reumatoidea, linfoma T cutáneo.

\section{Abstract}

Patients with RA are at increased risk for several disorders, including cancer, infection and cardiovascular diseases. Rates of hematological malignancies (i.e. lymphomas, myeloid leukaemia) are elevated in this patient population. This is a case of a 74 years old male patient with longstanding nodular and polyarticular RA, treated with etanercept and abatacept without efficacy, later rituximab $2 \mathrm{~g} /$ yr for 3 years, with very good response. Maintenance treatment: tofacitinib $5 \mathrm{mg} / \mathrm{d}$ orally. In april 2016, he presented nodular and erytemathous lesions in legs, necrotizing ulcers in left leg, bullous lesions in feet and edema in lower limbs to thigh. Treatment stops. Skin biopsy: T cell cutaneous lymphoma with dermal and hypodermic commitment. First chemotherapy cycle (CHOP) well tolerated but after second $\mathrm{CHOP}$ cycle the patient presented bone marrow aplasia and died.

Key words: rheumatoid arthritis, T cell cutaneous lymphoma.

\section{Introducción}

Los pacientes con artritis reumatoidea (AR) con o sin tratamiento inmunosupresor tienen un riesgo incrementado de desórdenes linfoproliferativos respecto a la población general. La mayoría de ellos linfomas de células B, con escasos reportes de linfomas de células T, en especial cutáneos ${ }^{1,2,3}$. La $\mathrm{AR}$, sobre todo en sus formas de presentación más severas con compromiso extraarticular o sistémico, suele ser sometida a diversos esquemas terapéuticos con inmunomoduladores, glucocorticoides y fármacos biológicos para lograr su remisión o al menos mejorar la evolución de la enfermedad para hacerla menos agresiva. Los agentes biológicos pueden ser muy útiles en el tratamiento de la AR. Las personas tratadas con estos fármacos requieren controles estrictos, ya que podrían predisponer a eventos como infecciones, ${ }^{4}$ reacciones de hipersensibilidad, neoplasias ${ }^{5} \mathrm{y}$, ocasionalmente, leucemias o linfomas como reportan los ensayos clínicos. Sin embargo, en datos de registros y metaanálisis se sabe que la tasa estandarizada de trastornos linfoproliferativos no es diferente a la de pacientes no expuestos a terapias biológicas ${ }^{6}$.

\section{Objetivo}

Reportar y compartir con la comunidad médica un caso inusual de Linfoma T cutáneo durante el curso de una artritis reumatoidea agresiva, estable, en tratamiento con fármacos biológicos de larga data.

\section{Caso clínico}

Paciente de sexo masculino de 74 años de edad, productor agropecuario, con antecedentes personales patológicos de HTA, lepra (tratada hace más de 30 años), AR nodular y 
erosiva de 25 años de evolución con compromiso severo poliarticular y extraarticular con nódulos reumatoideos en manos, codos y rodillas. Recibió tratamiento con etanercept y abatacept, suspendidos por falta de eficacia y rituximab en dosis anuales de 2 g/año por tres años consecutivos, que se inició por la importante nodulosis, con excelente respuesta, decidiendo continuar mantenimiento con tofacitinib $5 \mathrm{mg} /$ día vía oral (VO).

Es derivado a nuestro servicio en abril de 2016 por nódulos y úlceras en ambos miembros inferiores que habían comenzado dos meses antes, posteriormente se esfacelaron y comenzaron con secreción serosa, que luego se torna purulenta.

Se suspende tofacitinib y se indica antibioticoterapia, primero minociclina y luego cefadroxilo, sin respuesta. Al momento de la consulta a Reumatología presenta neumopatía con derrame pleural y peor estado de sus lesiones ulcerativas y nodulares. Por su tórpida evolución se decide internación para estudio y tratamiento.

Niega fiebre, artralgias, artritis pero refiere tos seca y disnea grado III desde varios meses atrás (antecedente de neumonía siete meses antes). Al examen físico se observan lesiones nodulares eritematosas de 2 a $5 \mathrm{~cm}$ en ambas piernas de consistencia renitente y úlceras en tercio inferior

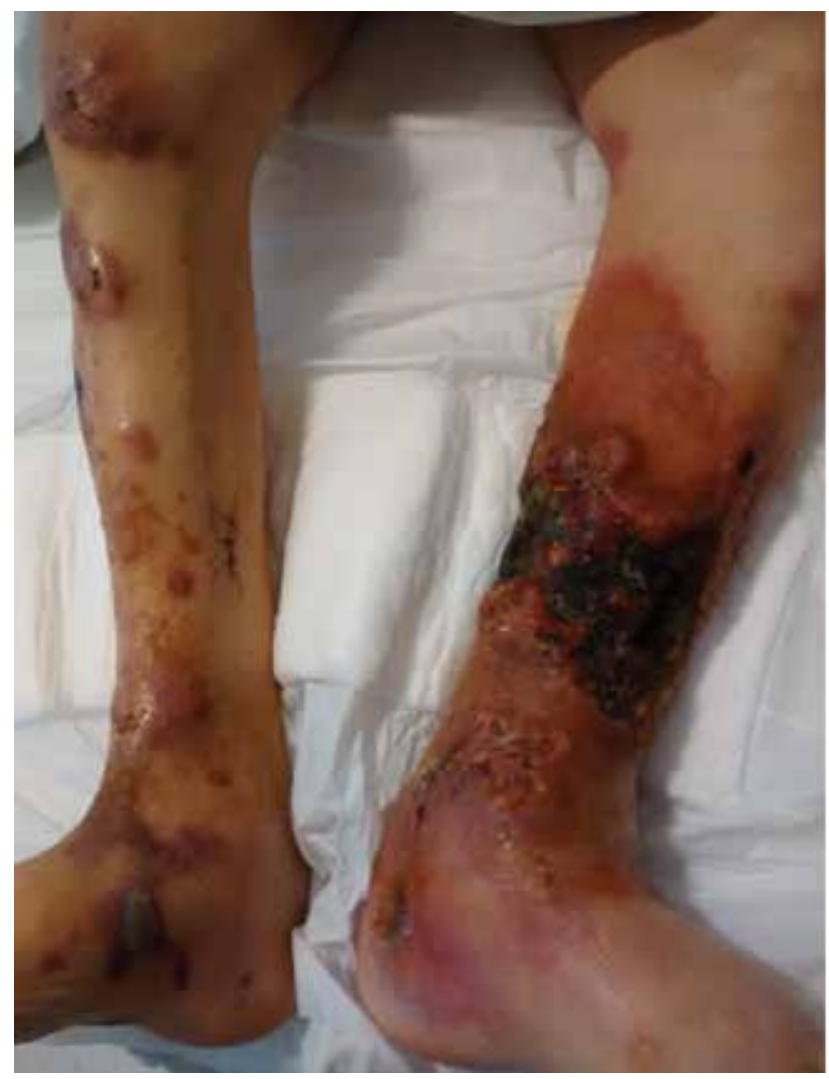

Figura 1. Lesiones nodulares eritematosas en ambas piernas y úlceras en tercio inferior de cara anterior e interna pierna izquierda fondo necrótico y úlcera en maléolo interno de pie izquierdo. Lesiones ampollares de contenido hemático en región interna de pie derecho y planta del pie izquierdo. Edema de miembro inferior izquierdo hasta raíz de muslo.

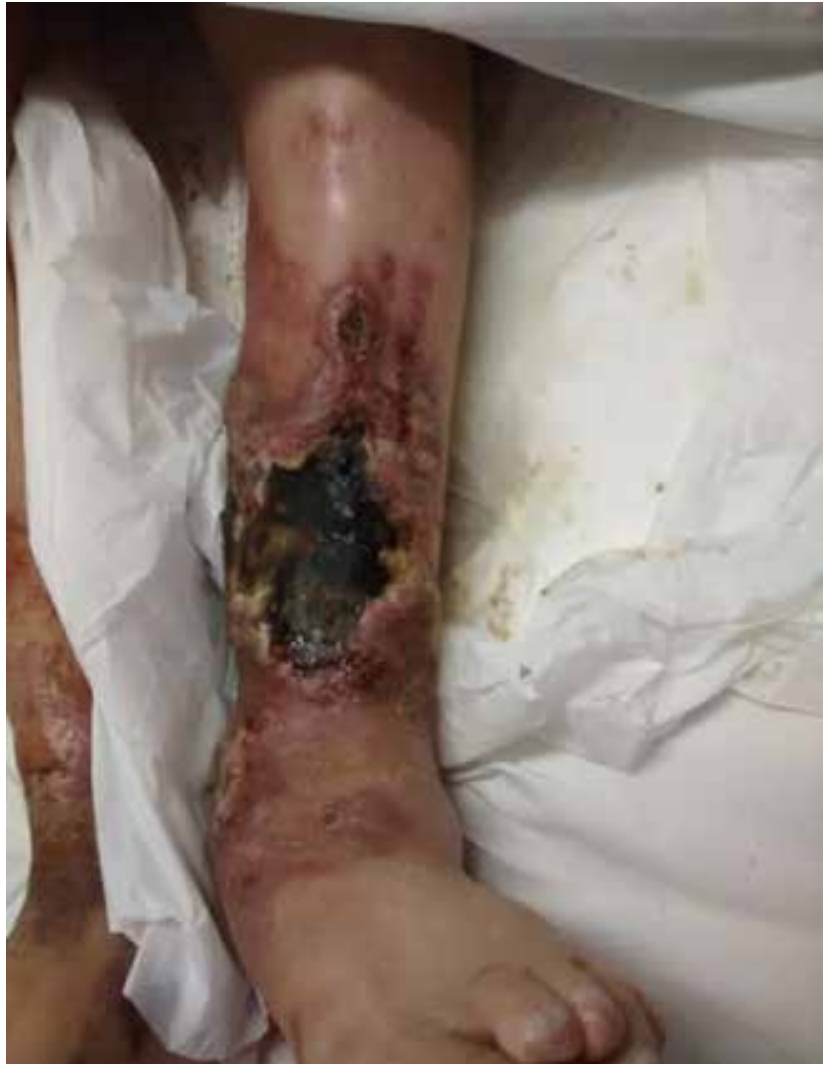

Figura 2. Evolución de las lesiones durante el tratamiento parenteral con antibioticoterapia.

de cara anterior e interna pierna izquierda de $10 \times 8 \mathrm{~cm}$, de bordes netos, fondo necrótico y secreción serosa. Otra úlcera de $3 \times 3 \mathrm{~cm}$ en maléolo interno de pie izquierdo. Lesiones ampollares de contenido hemático en región interna de pie derecho y planta del pie izquierdo. Edema de miembro inferior izquierdo hasta raíz de muslo. Laboratorio: Hb: 13 gr\%, Hto 41\%, leucocitos 6400/mm³ (NC:3/NS:75/L:15/M:3) VSG: 17, ASAT: 32 U/1, ALAT: 15 U/1, GGT: 29 U/1, FAL: $95 \mathrm{U} / 1, \mathrm{LDH}: 613 \mathrm{U} / 1$, bilirrubina total: $0,67 \mathrm{mg} \%$. Sedimento y físico-químico de orina normal. $R x$ de tórax: ocupación de senos costodiafragmáticos a predominio de izquierdo, infiltrado radiopaco en base pulmonar y campo medio de pulmón izquierdo. Hemocultivo, urocultivo negativos. El líquido pleural fue serosanguinolento sin células malignas. La tomografía computada (TC) tóraco-abdominal evidencia "derrame pleural bilateral con atelectasia subsegmentaria adyacente. En lóbulo inferior izquierdo y língula se observa engrosamiento reticular asociado a opacidad en vidrio esmerilado. No adenomegalias mediastinales. En abdomen: líquido libre de distribución multicompartimental, ligera hepatomegalia".

Comienza tratamiento antibiótico parenteral (ciprofloxacina + clindamicina) cubriendo ambos focos, partes blandas y pulmonar en paciente inmunodeprimido, de acuerdo a indicaciones del servicio de Infectología. Se realiza interconsulta con servicio de Dermatología, quienes descartan 


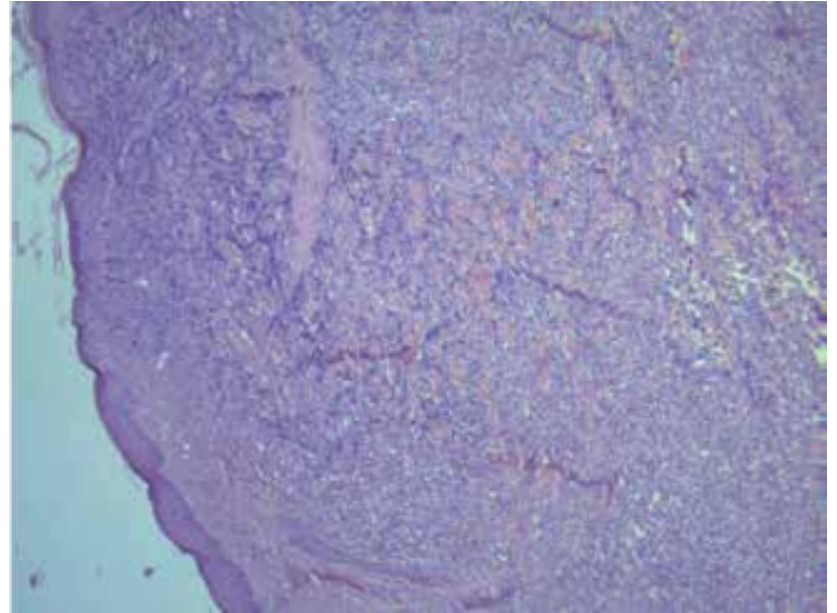

Figura 3. Piel con afectación dérmica por la proliferación linfoide. Hematoxilina Eosina (H/E) 50X.

compromiso lepromatoso según antecedentes previos y sospechan origen neoplásico de las lesiones, confirmado por anatomía patológica: piel con afectación dérmica por la proliferación de células neoplásicas de aspecto linfoide invasoras con afectación del tejido celular subcutáneo con marcación para CD3 (+); CD7 (+); CD8 (+); CD20 (-); CD30 (-); CD56 (-); VEB (-). Los hallazgos histológicos e inmunohistoquímicos son vinculables a un linfoma cutáneo de células T con compromiso dérmico e hipodérmico.

En mayo comienza primer ciclo de quimioterapia $\mathrm{CHOP}$ (ciclofosfamida, doxorrubicina, vincristina, prednisona) con buena tolerancia y mejoría de úlceras en miembros inferiores. En julio de 2016 fallece tras reingresar a esta institución con diagnóstico de pancitopenia secundaria a aplasia medular asociada a infección.

\section{Discusión}

La AR puede causar una variedad de lesiones dérmicas. Los nódulos subcutáneos ocurren en aproximadamente el $25 \%$ de los pacientes con artritis reumatoidea y es más común en los hombres. Pueden ocurrir complicaciones, como la infección o ulceración. La vasculitis es una manifestación extraarticular potencialmente seria de la $\mathrm{AR}^{7}$. Las lesiones se encuentran comúnmente en las piernas, pueden ulcerarse y ser dolorosas. La vasculitis puede conducir a la gangrena de la piel en el miembro afectado. El pioderma gangrenoso se puede presentar hasta en el 37\% de los pacientes con AR. Esto causa lesiones ulceradas que progresan rápidamente en tamaño, comúnmente en las extremidades inferiores. La dermatitis granulomatosa intersticial es otra lesión infrecuente de la piel, cuyos síntomas incluyen pápulas eritematosas ${ }^{8}$. Existen otras causas de compromiso dérmico en AR pero la más temida es la presencia de linfomas cutáneos, ya sean de la estirpe celular B o T con una frecuencia de aparición menor al $1 \% \%^{1,2}$.

La AR se caracteriza por estimulación inmune persistente, la cual podría llevar a proliferación linfocítica policlonal con el aumento potencial de transformación maligna. Los pacientes con AR, sobre todo aquellos con enfermedad más agresiva, ${ }^{9}$ tienen un riesgo aumentado (de 1,5 a 8,7 veces más) para desarrollo de linfoma, principalmente linfoma no Hodgkin, respecto de la población general ${ }^{10,11,12}$.

En un artículo reciente de revisión sobre los desencadenantes probables de este tipo de linfoma cutáneo, se propone al Mycobacterium leprae como uno de ellos. Es importante destacar que este paciente había sido tratado 30 años antes por padecer de lepra lepromatosa y no presentaba signos y síntomas de actividad de la enfermedad infecciosa ${ }^{13}$.

Los anti-TNF no parecerían aumentar la incidencia de linfoma en AR, aunque los datos basados en ensayos clínicos sugieren una asociación entre estos fármacos y cáncer. En ensayos clínicos con infliximab vs placebo, los índices de incidencia fueron $0,09 \%$ comparados con $0 \%$ para linfoma; en pacientes tratados con etanercept por más de 6 años, la incidencia de neoplasias fue $3 \%$, consistente con la que generalmente se observa en este grupo de pacientes; en grupos de adalimumab y certolizumab vs controles, el índice de eventos para 100 pacientes año (PA) fue 0,07 para linfoma. En pacientes tratados con golimumab vs placebo, el índice de eventos para 100 PA fue 0,1 vs 0 para linfoma respectivamente ${ }^{14,15}$.

Los pacientes tratados con anti-TNF tienen un riesgo aumentado de eventos infecciosos serios, con una incidencia tan alta como 0,07-0,09/PA vs 0,01-0,06/PA para otros tratamientos, siendo las infecciones de la piel, tejidos blandos $\mathrm{y}$ articulaciones las más frecuentes ${ }^{15}$.

\section{Conclusión}

La aparición de lesiones dérmicas en un paciente con AR bajo tratamiento prolongado y sobre todo con enfermedad controlada, debe alertarnos a pensar en complicaciones por inmunosupresión, entre ellas los linfomas dérmicos agresivos. El control y manejo interdisciplinario es fundamental y la anatomía patológica es una herramienta esencial para el manejo correcto de estas comorbilidades.

\section{Bibliografía}

1. Ekström K, Hjalgrim H, Brandt L, Baecklund E, Klareskog L, Ekbom A, et al. Risk of malignant lymphomas in patients with rheumatoid arthritis and in their first-degree relatives. Arth Rheum 2003; 48:963-70.

2. Georgescu L, Quinn GC, Schwartzman S, Paget SA. Lymphoma in patients with rheumatoid arthritis: association with the disease state or methotrexate treatment. Semin Arthritis Rheum 1997; 26:794-804.

3. Smitten AL, Simon TA, Hochberg MC, Suissa S. A metaanalysis of the incidence of malignancy in adult patients with rheumatoid arthritis. Arthritis Research \&Therapy 2008, 10:R45.

4. Thomason RW, Craig FE, Banks PM, Sears DL, Myerson GE, Gulley ML. Epstein-Barr virus and lymphoproliferation in methotrexate-treated rheumatoid arthritis. Mod Pathol 1996; 9:261-6.

5. Askling J, Fored CM, Brandt L, Baecklund E, Bertilsson 

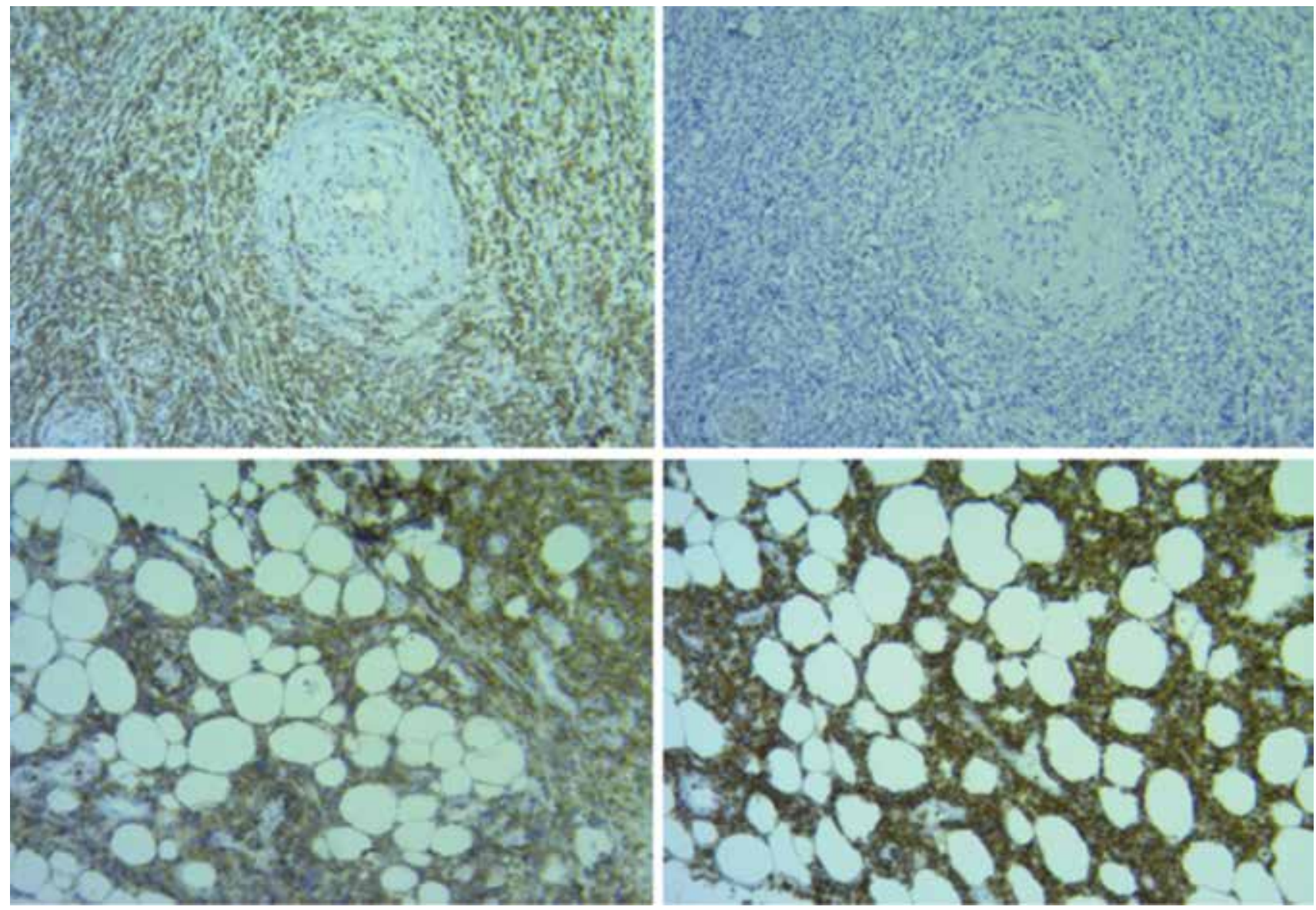

Figura 4. Marcación para CD3 (+) esquina superior izquierda; CD20 (-) esquina superior derecha; CD8 (+) esquina inferior izquierda; CD7 (+) esquina inferior derecha.

L, Feltelius N. Risks of solid cancers in patients with rheumatoid arthritis and after treatment with tumour necrosis factor antagonists. Ann Rheum Dis 2005; 64:1421-1426.

6. Mariette X, Tubach F, Bagheri H, Bardet M, Berthelot JM, Gaudin P, Heresbach D, Martin A, Schaeverbeke T, Salmon D, Lemann M, Hermine O, Raphael M, Ravaud P. Lymphoma in patients treated with anti-TNF: results of the 3-year prospective French RATIO registry. Ann Rheum Dis 2010; 69:400-408.

7. Toussirot É, Aubin F. RMD Open 2016.

8. Maldonado Cocco J, Citera G. et al. Reumatologia 2010. Ediciones Azzurras. Buenos Aires. pag 235-237.(245)

9. Cancer Epidemiol Biomarkers Prev 2006; 15 (11). November 2006

10. Baecklund E, Ekbom A, Sparen P, Feltelius N, Klareskog L. Disease activity and risk of lymphoma in patients with rheumatoid arthritis: Nested case-control study. Br Med J 1998; 317:180-181.

11. Baecklund E, Iliadou A, Askling J, Ekbom A, Backlin C, Granath F. Association of chronic inflammation, not its treatment, with increased lymphoma risk in rheumatoid arthritis. Arthritis Rheum 2006, 54:692-701.

12. Llorca J, López-Díaz MJ, González-Juanatey C, Ollier WER, Martin J, González-Gay MA. Persistent chronic inflammation contributes to the development of cancer in patients with rheumatoid arthritis from a defined population of northwestern Spain. Semin Arthritis Rheum 2007; 37:31-38.

13. Litvinov Ivan, Shtreis Anna, Kobayashi Kenneth, Glassman Steven, Tsang Matthew, Woetmann Anders, Sasseville Denis, Ødum Niels, Duvic Madeleine. Investigating potential exogenous tumor initiating and promoting factors for Cutaneous T-Cell Lymphomas (CTCL), a rare skin malignancy. Oncoimmunology 2016, VOL. 0, NO. 0, e1175799.

14. Askling J, Fored CM, Baecklund E, Brandt L, Backlin C, Ekbom A. Haematopoietic malignancies in rheumatoid arthritis: Lymphoma risk and characteristics after exposure to tumour necrosis factor antagonists. Ann Rheum Dis 2005; 64:1414-1420.

15. Rheumatology 2012; 51(suppl 5):v38-v47. 